\title{
Outer bounds on the error correction capacity region for non-multicast networks
}

\author{
Svitlana Vyetrenko \\ California Institute of Technology \\ Pasadena, CA 91125, USA \\ Email: svitlana@caltech.edu
}

\author{
Tracey Ho \\ California Institute of Technology \\ Pasadena, CA 91125, USA \\ Email: tho@caltech.edu
}

\author{
Theodoros K. Dikaliotis \\ California Institute of Technology \\ Pasadena, CA 91125, USA \\ Email: tdikal@caltech.edu
}

\begin{abstract}
In this paper we study the capacity regions of non-multicast networks that are susceptible to adversarial errors. We derive outer bounds on the error correction capacity region and give a family of single- and two-source two-sink 3-layer networks for which these bounds are tight.
\end{abstract}

\section{INTRODUCTION}

In this paper we consider the problem of adversarial error correction in non-multicast networks. The network error correction problem, where an adversary arbitrarily corrupts transmissions on an unknown set of $z$ links, was introduced by Cai and Yeung [1]. Previous work on network error correction largely assumes multicast network scenarios. For single- and multiple-source multicast network scenarios, it has been proven that the cutset bounds are tight, and that linear network error-correcting codes are sufficient [1], [2]. Various centralized and decentralized schemes that achieve these bounds are also known, e.g. [3], [4], [5].

For non-multicast networks, however, finding the capacity region of a general network even in the error-free case is an open problem. There exist simple examples of non-multicast networks, whose error-free capacity regions are not described by the cutset bounds or are not polyhedral [6], [7]. The capacity region of singlesource two-sink networks [8], [9], [10] as well as singlesource disjoint- or nested-demand networks [11] with any number of sinks is known to be described by the cutset bounds in the error-free case. In this paper we show that this is not the case for erroneous single-source two-sink networks. We provide upper bounds on the error correction capacity regions of non-multicast networks based on the topological structure of network cuts. We also show that our previous achievability construction

\footnotetext{
This work was supported in part by the Air Force Office of Scientific Research under grant FA9550-10-1-0166 and Caltech's Lee Center for Advanced Networking.
}

based on a given linear error-free achievable region in [2] is capacity-achieving for a family of single- and twosource two-sink 3-layer networks in the presence of network errors, and use this to provide a tighter upper bound for general two-sink networks.

The paper is organized as follows. In Section II, we introduce the notation used throughout the paper. In Section III, we review our achievability construction in the presence of errors based on the linear achievable region in the error-free case. In Sections IV and V respectively, we derive upper bounds on the error correction capacity regions for general multiple-sink and two-sink networks. In Section VI we provide examples to illustrate our results.

\section{MODEL}

Consider a network error correction problem on a directed acyclic graph $\mathcal{G}$ with $n$ source nodes $\mathcal{S}=$ $\left\{s_{1}, s_{2}, \ldots, s_{n}\right\}$ and $m$ sink nodes $\mathcal{T}=\left\{t_{1}, t_{2}, \ldots, t_{m}\right\}$, where each source $s_{i}$ is demanded by a given set of sink nodes $\mathcal{T}_{i}$, and arbitrary coding across sessions is permitted. Each link has unit capacity, and there can be multiple parallel edges connecting a pair of nodes.

For each $i, i \in\{1, \ldots, n\}$, let $r_{i}$ be the error-free information rate of $s_{i}$. For any non-empty subset $\mathcal{S}^{\prime} \subseteq \mathcal{S}$, let $\mathcal{I}\left(\mathcal{S}^{\prime}\right)$ be the indices of the source nodes that belong to $\mathcal{S}^{\prime}$. Similarly, for any non-empty subset of $\mathcal{T}^{\prime} \in \mathcal{T}$, let $\mathcal{I}\left(\mathcal{T}^{\prime}\right)$ be the indices of the sink nodes that belong to $\mathcal{T}^{\prime}$. Define $m_{\mathcal{S}^{\prime}, \mathcal{T}^{\prime}}$ to be the minimum cut capacity between $\mathcal{S}^{\prime}$ and $\mathcal{T}^{\prime}$.

A network code is $z$-error link-correcting if it can correct any $t$ adversarial link errors for $t \leq z$. For each $i, i \in$ $\{1, \ldots, n\}$, let $u_{i}$ be the information rate of $s_{i}$ in case of any $z$ network link errors. The set of all rate vectors $\left(u_{1}, u_{2}, \ldots, u_{n}\right)$ that can be achieved on $\mathcal{G}$ under any $z$ 
network link errors is called $z$-error correction capacity region.

Define $\phi_{l}(\mathbf{x})$ as the error-free output of link $l$ when the network input is $\mathbf{x} \in \mathcal{C}$. If an error vector $\mathbf{z}$ occurs, its components are added to the link inputs according to the coding order. Then the output of a link $l$ is a function of both the network input $\mathbf{w}$ and the error vector $\mathbf{z}$ and it is denoted by $\psi_{l}(\mathbf{w}, \mathbf{z})[1]$.

Throughout the paper, we assume the coherent network coding scenario, in which there is centralized knowledge of the network topology and network code.

\section{ACHIEVABILITY CONSTRUCTION}

In [2] we proved the following achievability result for the coherent case:

Theorem 1: Given any linear network code $\mathcal{C}$ that achieves rate vector $\mathbf{r}=\left(r_{1}, r_{2}, \ldots, r_{n}\right)$ in the errorfree case, where $r_{i}$ is the information rate of source $s_{i}, i=1, \ldots, n$, we can obtain a network code $\tilde{\mathcal{C}}$ that achieves rate vector $\tilde{\mathbf{r}}=\left(r_{1}-2 z, r_{2}-2 z, \ldots, r_{n}-2 z\right)$ under arbitrary errors on up to $z$ links in the network.

Let $\mathbb{C} \subseteq \mathbb{R}^{n}$ be an error-free region achievable in $\mathcal{G}$ by linear coding. Then Theorem 1 allows us to construct an achievable error-correction region $\mathbb{V}$ based on $\mathbb{C}$ as follows:

- Take any achievable rate vector $\mathbf{r}=$ $\left(r_{1}, r_{2}, \ldots, r_{n}\right) \in \mathbb{C}$

- Define $f(\mathbf{r})=\left(\max \left(r_{1}-2 z, 0\right), \ldots, \max \left(r_{n}-2 z, 0\right)\right)$.

- By Theorem 1, $f(\mathbf{r}) \in \mathbb{V}$.

- By timesharing, for any $0 \leq \lambda \leq 1$ and any $\mathbf{v}, \mathbf{w} \in$ $\mathbb{V}, \lambda \mathbf{v}+(1-\lambda) \mathbf{w} \in \mathbb{V}$.

In accordance with the above described procedure, we define

$$
\mathbb{A}=\left\{\mathbf{a} \in \mathbb{R}^{n}: \exists \mathbf{r} \in \mathbb{C} \text { such that } \mathbf{a}=f(\mathbf{r})\right\}
$$

to be the set of rate vectors that have a preimage in $\mathbb{C}$. Also define

$$
\begin{aligned}
\mathbb{T}= & \left\{\mathbf{t} \in \mathbb{R}^{n} \backslash \mathbb{A}: \exists k_{1}, k_{2}, \ldots, k_{n}, \sum_{i=1}^{n} k_{i}=n\right. \\
& \left.\mathbf{r}_{1}, \mathbf{r}_{2}, \ldots, \mathbf{r}_{n} \in \mathbb{A} \text { such that } \mathbf{t}=\sum_{i=1}^{n} \frac{k_{i}}{n} \mathbf{r}_{i}\right\}
\end{aligned}
$$

to be the set of rate vectors that can be achieved under any $z$ errors by timesharing of elements in $\mathbb{A}$. Note that by our construction $\mathbb{V}=\mathbb{A} \cup \mathbb{T}$.

Figure 1 illustrates the construction of $\mathbb{V}$ based on $\mathbb{C}$
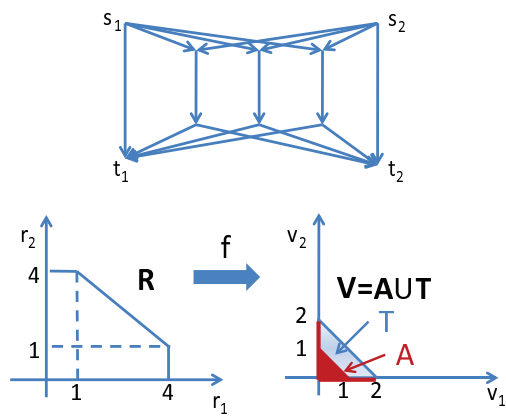

Fig. 1. Example of the error correction achievability construction based on the known error-free capacity region for the two-source twosink network.

for the two-source two-sink network, for which in the error-free case the cutset bounds

$$
\begin{aligned}
r_{1} & \leq m_{s_{1}, t_{1}}=4 \\
r_{2} & \leq m_{s_{2}, t_{2}}=4 \\
r_{1}+r_{2} & \leq m_{s_{1} s_{2}, t_{1} t_{2}}=5
\end{aligned}
$$

are achieved.

\section{UPPER BOUND FOR GENERAL NETWORKS}

In this section we consider an acyclic network $\mathcal{G}=$ $(V, E)$ with source set $\mathcal{S}$ and sink set $\mathcal{T}=$ $\left\{t_{1}, t_{2}, \ldots, t_{m}\right\}$. Let $X_{1}, X_{2}, \ldots, X_{m}$ be $m$ independent source processes, such that each $X_{i}$ is demanded by exactly one $t_{i}$ (we require non-overlapping sink demands). Let $\mathcal{C}=\mathcal{C}_{1} \times \mathcal{C}_{2} \times \ldots \times \mathcal{C}_{m}$ be the code used by $\mathcal{S}$.

Define $P=\left(V_{\mathcal{S}}, V_{\mathcal{T}}\right)$ to be a partition of $V$ such that all sources are in $V_{\mathcal{S}}$ and all sinks are in $V_{\mathcal{T}}$. Define

$$
\operatorname{cut}(P)=\left\{(a, b) \in E \mid a \in V_{\mathcal{S}}, b \in V_{\mathcal{T}}\right\} .
$$

Further, for any non-empty subset $T^{\prime} \subseteq \mathcal{T}$ define

$$
L_{T^{\prime}}^{P}=\left\{e \in \operatorname{cut}(P): e \text { is upstream of all } t \in T^{\prime}\right.
$$
and $e$ is not upstream of any $\left.t \in \mathcal{T} \backslash T^{\prime}\right\}$.

Note that for any $T^{\prime}, T^{\prime \prime} \subseteq \mathcal{T}$ such that $T^{\prime} \neq T^{\prime \prime}, L_{T^{\prime}}^{P} \cap$ $L_{T^{\prime \prime}}^{P}=\emptyset$, therefore,

$$
|\operatorname{cut}(P)|=\sum_{T^{\prime} \subseteq \mathcal{T}}\left|L_{T^{\prime}}^{P}\right|
$$

Similar to [12], [13], we use the following definition: 
Definition 1: A subset of links $Q \subseteq \operatorname{cut}(P)$ is said to satisfy the downstream condition (DC) if none of the remaining links in $\operatorname{cut}(P)$ are downstream of any link in $Q$.

Let $\mathbb{U}=\left\{\left(u_{1}, u_{2}, \ldots, u_{m}\right)\right\}$ denote the $z$-error correction capacity region of $\mathcal{G}$. In Theorem 2 , we derive an upper bound on $\mathbb{U}$ by considering an optimization that chooses subsets $S_{T^{\prime}}^{P}$ of each set $L_{T^{\prime}}^{P}$ of links on $\operatorname{cut}(P)$ such that the union of the chosen subsets satisfies DC and at most $2 z$ chosen links are upstream of each sink.

Theorem 2: For any $\left(u_{1}, u_{2}, \ldots, u_{m}\right) \in \mathbb{U}$

$$
\sum_{j=1}^{m} u_{j} \leq \min _{P=\left(V_{\mathcal{S}}, V_{\mathcal{T}}\right)}\left(|\operatorname{cut}(P)|-l^{P}\right),
$$

where $l^{P}$ is a solution to

$$
\begin{aligned}
& \text { maximize } l^{P}=\sum_{T^{\prime} \subseteq \mathcal{T}}\left|S_{T^{\prime}}^{P}\right| \\
& \text { subject to } \\
& \forall t_{i} \in \mathcal{T} \quad \sum_{T^{\prime} \subseteq \mathcal{T}: t_{i} \in T^{\prime}}\left|S_{T^{\prime}}^{P}\right| \leq 2 z \\
& \forall T^{\prime} \subseteq \mathcal{T} \quad\left|S_{T^{\prime}}^{P}\right| \leq\left|L_{T^{\prime}}^{P}\right| \\
& \bigcup_{T^{\prime} \subseteq \mathcal{T}: t_{i} \in T^{\prime}} S_{T^{\prime}}^{P} \text { satisfies DC. }
\end{aligned}
$$

Proof: We prove the statement of this theorem by contradiction. Suppose there exists $\left(u_{1}^{*}, u_{2}^{*}, \ldots, u_{m}^{*}\right) \in$ $\mathbb{U}_{1, m}$ such that for some $P=\left(V_{\mathcal{S}}, V_{\mathcal{T}}\right)$

$$
\sum_{i=j}^{m} u_{j}^{*}>M-l^{P} .
$$

For notational convenience let $|\operatorname{cut}(P)|=M$ and denote the links in $\operatorname{cut}(P)$ by $\left\{a_{1}, a_{2}, \ldots, a_{m}\right\}$ indexed in increasing topological order. By (6), for any $M-l^{p}$ links there exist two codewords $\mathbf{x}=\left(\mathbf{x}_{1}, \mathbf{x}_{2}, \ldots, \mathbf{x}_{m}\right)$ and $\mathbf{y}=\left(\mathbf{y}_{1}, \mathbf{y}_{2}, \ldots, \mathbf{y}_{m}\right)$ in $\mathcal{C}, \mathbf{x} \neq \mathbf{y}$, such that $\phi_{a_{f}}(\mathbf{x})=\phi_{a_{f}}(\mathbf{y})$ for $M-l^{p}$ indexes $a_{f}$. Note that by (1) and (2), the set $\operatorname{cut}(P) \backslash\left(\bigcup_{T^{\prime} \subseteq \mathcal{T}} S_{T^{\prime}}^{P}\right)$ has size

$$
M-l^{P}=\sum_{T^{\prime} \subseteq \mathcal{T}}\left(\left|L_{T^{\prime}}^{P}\right|-\left|S_{T^{\prime}}^{P}\right|\right),
$$

therefore, by (6) we can choose $\mathbf{x}$ and $\mathbf{y}$ so that

$$
\phi_{a_{f}}(\mathbf{x})=\phi_{a_{f}}(\mathbf{y}), a_{f} \in \operatorname{cut}(P) \backslash\left(\bigcup_{T^{\prime} \subseteq \mathcal{T}} S_{T^{\prime}}^{P}\right)
$$

Since $\mathbf{x} \neq \mathbf{y}$, there exists at least one index $i \in$ $\{1, \ldots, m\}$ such that $\mathbf{x}_{i} \neq \mathbf{y}_{i}$. We will now demonstrate that if (6) holds, then there exists an adversarial error pattern such that $t_{i}$ will not be able to distinguish between $\mathbf{x}$ and $\mathbf{y}$. Define $L_{I}=\bigcup L_{T^{\prime}}^{P}$ to be the subset $T^{\prime} \subseteq \mathcal{T}: t_{i} \in T^{\prime}$

of links of $\operatorname{cut}(P)$ upstream of $t_{i}$ and let $I=\left|L_{I}\right|$. By (7), $\mathbf{x}$ and $\mathbf{y}$ were chosen so that $\phi_{a_{f}}(\mathbf{x})=\phi_{a_{f}}(\mathbf{y})$ in at least $J=I-\sum_{T^{\prime} \subseteq \mathcal{T}: t_{i} \in T^{\prime}}\left|S_{T^{\prime}}^{P}\right|$ positions. By constraint (3), $J \geq I-2 z$.

Define the error-free output of the links in $L_{I}$ by

$$
O(\mathbf{x})=\left\{\phi_{f_{1}}(\mathbf{x}), \phi_{f_{2}}(\mathbf{x}), \ldots, \phi_{f_{I}}(\mathbf{x})\right\},
$$

where all links $f_{l} \in L_{I}$ and $\phi_{f_{l}}($.$) are indexed in the$ increasing coding order. Hence, by (5) and (7) we can write

$$
\begin{aligned}
& O(\mathbf{x})=\left\{x_{1}, x_{2}, \ldots, x_{J}, x_{J+1}^{\prime}, \ldots, x_{I}^{\prime}\right\} \\
& O(\mathbf{y})=\left\{x_{1}, x_{2}, \ldots, x_{J}, x_{J+1}^{\prime \prime}, \ldots, x_{I}^{\prime \prime}\right\}
\end{aligned}
$$

Assume the network input is $\mathbf{x}$. The adversary will inject $z$ error symbols $\mathbf{z}_{x}=\left(z_{x_{1}}, z_{x_{2}}, \ldots, z_{x_{z}}\right)$ on links $a_{f_{J+1}}, \ldots, a_{f_{J+z}}$ as follows. First it injects $z_{x_{1}}$ on link $a_{f_{J+1}}$ so that

$$
\psi_{a_{f_{J+1}}}\left(\mathbf{x},\left(z_{x_{1}}, 0,0, \ldots, 0\right)\right)=x_{J+1}^{\prime \prime} .
$$

Then the output of links $a_{f_{J+2}}, \ldots, a_{f_{I}}$ is affected, but not of $a_{f_{1}}, \ldots, a_{f_{J}}$. With this consideration, next the adversary injects the symbols $z_{x_{2}}$ on link $a_{f_{J+2}}$ so that

$$
\psi_{a_{f_{J+2}}}\left(\mathbf{x},\left(z_{x_{1}}, z_{x_{2}}, 0 \ldots, 0\right)\right)=x_{J+2}^{\prime \prime} .
$$

The output of links $a_{f_{J+3}}, \ldots, a_{f_{I}}$ is affected, but not of $a_{f_{1}}, \ldots, a_{f_{J+1}}$. The process continues until the adversary finishes injecting $z$ errors at links $a_{J+1}, \ldots, a_{J+z}$. Let $E(\mathbf{x}, \mathbf{z})=\left\{\psi_{a_{f_{1}}}(\mathbf{x}, \mathbf{z}), \ldots, \psi_{a_{f_{I}}}(\mathbf{x}, \mathbf{z})\right\}$, then

$$
\begin{aligned}
E\left(\mathbf{x}, \mathbf{z}_{x}\right)= & \left\{x_{1}, \ldots, x_{J}, x_{J+1}^{\prime \prime}, \ldots, x_{J+z}^{\prime \prime}\right. \\
& \left.x_{J+z+1}^{\prime \prime \prime}, \ldots, x_{I}^{\prime \prime \prime}\right\} .
\end{aligned}
$$

Now suppose the network input is $\mathbf{y}$. The adversary will inject $z$ error symbols $\mathbf{z}_{y}=\left(z_{y_{1}}, z_{y_{2}}, \ldots, z_{y_{z}}\right)$ on links $a_{f_{J+z+1}}, \ldots, a_{f_{I}}$ as follows. First it injects $z_{y_{1}}$ on link $a_{f_{J+z+1}}$ so that

$$
\psi_{a_{f_{J+z+1}}}\left(\mathbf{x},\left(z_{y_{1}}, 0,0, \ldots, 0\right)\right)=x_{J+z+1}^{\prime \prime \prime} .
$$

Then the output of links $a_{f_{J+z+2}}, \ldots, a_{f_{I}}$ is affected, but not of $a_{f_{1}}, \ldots, a_{f_{J+z}}$. With this consideration, next the adversary injects the symbols $z_{y_{2}}$ on link $a_{f_{J+z+2}}$ so that

$$
\psi_{a_{f+z+2}}\left(\mathbf{x},\left(z_{y_{1}}, z_{y_{2}}, 0 \ldots, 0\right)\right)=x_{J+2+1}^{\prime \prime \prime} .
$$

The output of links $a_{f_{J+z+3}}, \ldots, a_{f_{I}}$ is affected, but not of $a_{f_{1}}, \ldots, a_{f_{J+z+1}}$. Similarly, the process continues 
until the adversary finishes injecting at most $z$ errors at links $a_{J+z+1}, \ldots, a_{I}$. Then

$$
\begin{aligned}
E\left(\mathbf{x}, \mathbf{z}_{y}\right)= & \left\{x_{1}, \ldots, x_{J}, x_{J+1}^{\prime \prime}, \ldots, x_{J+z}^{\prime \prime}\right. \\
& \left.x_{J+z+1}^{\prime \prime \prime}, \ldots, x_{I}^{\prime \prime \prime}\right\} .
\end{aligned}
$$

Therefore, since $t_{i}$ is upstream of links only in $L_{I}$, it can observe only $E\left(\mathbf{x}, \mathbf{z}_{x}\right)$ and $E\left(\mathbf{y}, \mathbf{z}_{y}\right)$, hence, it would not be able to distinguish between $\mathbf{x}$ and $\mathbf{y}$.

Thus, for any $P=\left(V_{\mathcal{S}}, V_{\mathcal{T}}\right)$

$$
\sum_{j=1}^{m} u_{j}^{*} \leq|\operatorname{cut}(P)|-l^{P},
$$

therefore,

$$
\sum_{j=1}^{m} u_{j} \leq \min _{P=\left(V_{\mathcal{S}}, V_{\mathcal{T}}\right)}\left(|\operatorname{cut}(P)|-l^{P}\right) .
$$

\section{TWO-SINK NETWORKS}

In this section we consider any acyclic network $\mathcal{G}=$ $(V, E)$ with source set $\mathcal{S}$ and sink set $\mathcal{T}=\left\{t_{1}, t_{2}\right\}$ that demand independent (nonoverlapping) source processes. For cuts with no feedback links we derive a cut set bound that is tighter than the bound of Theorem 2. We also describe a family of 3-layer networks for which the bound is tight.

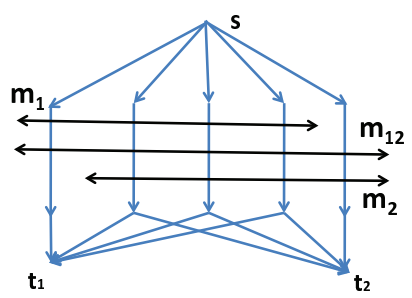

Fig. 2. Related 3-layer network.

As in the previous section, denote the $z$-error correction capacity region of $\mathcal{G}$ by $\mathbb{U}=\left\{u_{1}, u_{2}, \ldots, u_{m}\right\}$. From now on, for simplicity of notation, denote $m_{1} \doteq m_{s, t_{1}}$, $m_{2} \doteq m_{s, t_{2}}, m_{12} \doteq m_{s, t_{1} t_{2}}, L_{1}^{P} \doteq L_{t_{1}}^{P}, L_{2}^{P} \doteq L_{t_{2}}^{P}$ and $L_{12}^{P} \doteq L_{t_{1} t_{2}}^{P}$.

Definition 2: Let $P=\left(V_{s}, V_{\mathcal{T}}\right)$ be a partition of $\mathcal{G}$ that contains no feedback links. We construct a related onesource two-sink 3-layer network $\mathcal{G}_{1,2}^{3}(P)$ as follows. For each link $l$ in $\operatorname{cut}(P)$, we connect the source $s$ directly to the start node of $l$, and, for $i=1,2$, we connect the end node of $l$ directly to sink $t_{i}$ if $t_{i}$ is downstream of $l$ in the original network. This is illustrated in Figure 2).

Note that the construction of the related 3-layer network $\mathcal{G}_{1,2}^{3}(P)$ essentially allows all nodes on the source side of the cut to cooperate perfectly, and gives each sink at least as much information as it receives in the original network $\mathcal{G}$. Thus, the $z$-error correction capacity region $\mathbb{U}_{1,2}^{3}(P)$ of $\mathcal{G}_{1,2}^{3}(P)$ is an outer bound on the $z$-error correction capacity region $\mathbb{U}$ of $\mathcal{G}$.

In Section III we described how to construct an achievable error-correction region $\mathbb{V}_{1,2}^{3}(P) \subseteq \mathbb{U}_{1,2}^{3}(P)$ of $\mathcal{G}_{1,2}^{3}(P)$ based on its known error-free capacity region.

Lemma 1: The achievable region $\mathbb{V}_{1,2}^{3}(P)$ of the 3layer network $\mathcal{G}_{1,2}^{3}(P)$ is given by real-valued rate pairs $\left(u_{1}, u_{2}\right)$ such that

$$
\begin{array}{ll} 
& u_{1} \leq m_{1}-2 z \\
& u_{2} \leq m_{2}-2 z \\
& u_{1}\left(m_{2}-2 z-\max \left(m_{12}-m_{1}-2 z, 0\right)\right) \\
+ & u_{2}\left(m_{1}-2 z-\max \left(m_{12}-m_{2}-2 z, 0\right)\right) \\
\leq & \left(m_{1}-2 z\right)\left(m_{2}-2 z\right) \\
- & \max \left(m_{12}-m_{1}-2 z, 0\right) \max \left(m_{12}-m_{2}-2 z, 0\right)
\end{array}
$$

Proof: Since $\mathcal{G}_{1,2}^{3}(P)$ is a one-source two-sink network with nonoverlapping demands, its error-free capacity region is given by the cut set bounds [11] and achieved by time sharing among the rate pairs $(0,0),\left(m_{1}, 0\right),\left(m_{1}, m_{12}-m_{1}\right),\left(m_{12}-m_{2}, m_{2}\right)$ and $\left(0, m_{2}\right)$. Since this capacity region is polyhedral, by construction $\mathbb{V}_{1,2}^{3}(P)$ is also polyhedral and the corner points of $\mathbb{V}_{1,2}^{3}(P)$ are given by $(0,0),\left(m_{1}-2 z, 0\right)$, $\left(m_{1}-2 z, \max \left(m_{12}-m_{1}-2 z, 0\right)\right),\left(\max \left(m_{12}-m_{2}-\right.\right.$ $\left.2 z, 0), m_{2}-2 z\right)$ and $\left(0, m_{2}-2 z\right)$. Constraints (8)-(10) correspond to the lines joining the corner points of $\mathbb{V}_{1,2}^{3}(P)$.

Theorem 3: The z-error correction capacity region of any one-source two-sink 3-layer network $\mathcal{G}_{1,2}^{3}(P)$ is given by

$$
\mathbb{U}_{1,2}^{3}(P)=\mathbb{V}_{1,2}^{3}(P) .
$$

Proof: Since $\mathbb{V}_{1,2}^{3}(P)$ is achievable, it remains to show that

$$
\mathbb{U}_{1,2}^{3}(P) \subseteq \mathbb{V}_{1,2}^{3}(P) .
$$

Consider any point $\left(u_{1}, u_{2}\right) \in \mathbb{U}_{1,2}^{3}(P)$. By applying the cutset bounds for each sink individually, (8) and (9) are satisfied for $\left(u_{1}, u_{2}\right)$. Now we show that for any $\left(u_{1}, u_{2}\right) \in \mathbb{U}_{1,2}^{3}(P),(10)$ is also satisfied. 
- Case 1.

$$
\begin{aligned}
& m_{12}-m_{2} \geq 2 z \\
& m_{12}-m_{1} \geq 2 z .
\end{aligned}
$$

If (11) and (12) are satisfied, then (10) can be simplified as

$$
u_{1}+u_{2} \leq m_{12}-4 z .
$$

Note that (11) and (12) imply that

$$
\begin{aligned}
& \left|L_{1}^{P}\right| \geq 2 z \\
& \left|L_{2}^{P}\right| \geq 2 z .
\end{aligned}
$$

Then $l^{P}=4 z$ solves (2)-(5) with respect to partition $P$ and by Theorem 2

$$
u_{1}+u_{2} \leq m_{12}-4 z,
$$

which matches (13).

- Case 2.

$$
\begin{aligned}
& m_{12}-m_{2} \geq 2 z \\
& m_{12}-m_{1}<2 z
\end{aligned}
$$

If (14) and (15) are satisfied, then (10) can be simplified as

$$
\begin{aligned}
& \left(m_{2}-2 z\right) u_{1}+\left(m_{1}+m_{2}-m_{12}\right) u_{2} \\
\leq \quad & \left(m_{1}-2 z\right)\left(m_{2}-2 z\right) .
\end{aligned}
$$

Inequalities (14) and (15) imply that

$$
\begin{aligned}
m_{1} & \geq m_{2} \\
m_{1}-2 z & \geq m_{1}+m_{2}-m_{12} \\
m_{2}-2 z & <m_{1}+m_{2}-m_{12} .
\end{aligned}
$$

Denote the random process that is demanded by $t_{1}$ by $A$ and the random process demanded by $t_{2}$ by $B$. Index the links in $\operatorname{cut}(P)$ by $1,2, \ldots, m_{12}$, so that links $1, \ldots, m_{12}-m_{2}$ belong to $L_{1}^{P}$, links $m_{12}-m_{2}+1, \ldots, m_{1}$ belong to $L_{12}^{P}$, and links $m_{1}+1, \ldots, m_{12}$ belong to $L_{2}^{P}$. Note that in order for $t_{1}$ to decode, the symbols transmitted on any set of $m_{1}-2 z$ links upstream of $t_{1}$ must be distinct for every value of $A$ (in particular, on links $2 z+1, \ldots, m_{1}$ ). Similarly, in order for $t_{2}$ to decode, the symbols transmitted on any set of $m_{2}-2 z$ links upstream of $t_{2}$ must be distinct for every value of $B$ (in particular, this should hold for any subset of size $m_{2}-2 z$ of the shared links $\left.m_{12}-m_{2}+1, \ldots, m_{1}\right)$.

For simplicity of notation let

$$
\begin{aligned}
N & =m_{1}+m_{2}-m_{12} \\
K & =m_{2}-2 z \\
M & =\left(\begin{array}{c}
N \\
K
\end{array}\right) .
\end{aligned}
$$

Then, $\mathbb{U}_{1,2}^{3}(P)$ is upper-bounded by the error-free capacity region $\mathbb{U}^{\prime}=\left\{\left(u_{1}, u_{2}\right)\right\}$ of a one-source $(M+1)$-sink 3-layer network $\mathcal{G}^{\prime}$ constructed so that:

- Links $2 z+1, \ldots, m_{1}$ comprise the second layer.

- One sink $t^{\prime}$ is connected by $m_{1}-2 z$ links to all links in the second layer and demands process $A$. Each one of the remaining $M$ sinks is connected by $K$ links to a distinct size- $K$ subset of links $m_{12}-m_{2}+1, \ldots, m_{12}$ in the second layer and demands process $B$.

Further note that links $2 z+1, \ldots, m_{12}-m_{2}$ are connected to $t^{\prime}$, but not the other sinks of $\mathcal{G}^{\prime \prime}$. Hence, in order to upper bound the error-free capacity region on $\mathcal{G}^{\prime}$, we now can consider the error-free capacity region $\mathbb{U}^{\prime \prime}=\left\{\left(\tilde{u}_{1}, u_{2}\right)\right\}$ with $\tilde{u}_{1}=u_{1}-\left(m_{12}-m_{2}-2 z\right)$ of a one-source $(M+1)$-sink 3-layer network 3-layer network $\mathcal{G}^{\prime \prime}$ constructed so that:

- All $N$ links $m_{12}-m_{2}+1, \ldots, m_{1}$ from $L_{12}^{P}$ comprise the second layer.

- One sink $t_{A}^{\prime}$ is connected by $N$ links to all links in the second layer and demands process $A^{\prime}$, where $H\left(A^{\prime}\right)=H(A)-\left(m_{12}-\right.$ $\left.m_{2}-2 z\right)$. Each one of the remaining $M$ sinks $\left\{t_{B_{1}}, t_{B_{2}}, \ldots, t_{B_{M}}\right\}$ is connected by $K$ links to a distinct subset of all $N$ links in the second layer and demands process $B$.

An illustration of the above construction is given in Figure 3 for the case when $m_{1}=6, m_{2}=4$, $m_{12}=7$.

Denote the random processes transmitted on each

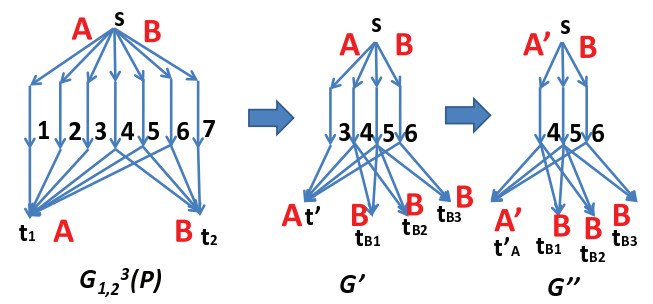

Fig. 3. Graphical illustration of the proof of Theorem 3 for the case when $m_{1}=6, m_{2}=4, m_{12}=7$.

one of the $N$ links $m_{12}-m_{2}+1, \ldots, m_{1}$ by $X^{N}=\left\{X_{1}, X_{2}, \ldots, X_{N}\right\}$. Let $\mathcal{S}_{K}$ be the set of all lexicographically ordered $K$-element subsets of $\{1, \ldots, N\}$. For any $\sigma \in \mathcal{S}_{K}$, let $\sigma(i)$ be the $i$ th element of $\sigma$. Let $\left\{X_{\sigma_{1}}, X_{\sigma_{2}}, \ldots, X_{\sigma_{M}}\right\}$ be the set of all unordered subsets of $X^{N}$ of size $K$. Then for any $\sigma \in \mathcal{S}_{K}$ we have

$$
I\left(A, B ; X_{\sigma}\right) \leq K
$$


Note that by the chain rule

$$
\begin{aligned}
I\left(A, B ; X_{\sigma}\right) & =I\left(A ; X_{\sigma}\right)+I\left(B ; X_{\sigma} \mid A\right) \\
& =I\left(A ; X_{\sigma}\right)+u_{2},
\end{aligned}
$$

therefore,

$$
I\left(A ; X_{\sigma}\right)+u_{2} \leq K
$$

and

$$
\sum_{\sigma \in \mathcal{S}_{K}} I\left(A ; X_{\sigma}\right)+M u_{2} \leq M K .
$$

Multiply this inequality by $\frac{N}{M}$, we get

$$
\frac{N}{M} \sum_{\sigma \in \mathcal{S}_{K}} I\left(A ; X_{\sigma}\right)+N u_{2} \leq N K .
$$

We now want to demonstrate that

$$
\frac{N}{M} \sum_{\sigma \in \mathcal{S}} I\left(A ; X_{\sigma}\right) \geq K \tilde{u_{1}}=K I\left(A ; X^{N}\right) .
$$

Note that

$$
\begin{aligned}
& I\left(A ; X_{\sigma} \mid B\right)-I\left(A ; X_{\sigma}\right) \\
= & H(A \mid B)-H\left(A \mid X_{\sigma}, B\right)-H(A)+H\left(A \mid X_{\sigma}\right) \\
= & H(A \mid B)-H\left(A \mid X_{\sigma}\right)-H(A)+H\left(A \mid X_{\sigma}\right) \\
= & 0,
\end{aligned}
$$

where the second equality follows since $B$ is a function of $X_{\sigma}$ and the third equality follows from independence of $A$ and $B$. Similarly, we can show that $I\left(A ; X^{N} \mid B\right)-I\left(A ; X^{N}\right)=0$, therefore, (18) can be rewritten as

$$
\frac{N}{M} \sum_{\sigma \in \mathcal{S}} I\left(A ; X_{\sigma} \mid B\right) \geq K I\left(A ; X^{N} \mid B\right) .
$$

Now notice that since $X_{\sigma}$ is a function of $A$ and $B$,

$$
\begin{aligned}
I\left(A ; X_{\sigma} \mid B\right) & =H\left(X_{\sigma} \mid B\right)-H\left(X_{\sigma} \mid A, B\right) \\
& =H\left(X_{\sigma} \mid B\right)
\end{aligned}
$$

and similarly $I\left(A ; X^{N} \mid B\right)=H\left(X^{N} \mid B\right)$. Then (19) is equivalent to

$$
N \sum_{\sigma \in \mathcal{S}_{K}} H\left(X_{\sigma} \mid B\right) \geq K M H\left(X^{N} \mid B\right) .
$$

By expanding the left- and right-hand sides of (20) using the chain rule, we get

$$
\begin{aligned}
& N \sum_{\sigma \in \mathcal{S}_{K}} \sum_{i=1}^{K} H\left(X_{\sigma(i)} \mid X_{\sigma(1)}, \ldots, X_{\sigma(i-1)}, B\right) \\
& \geq M K \sum_{i=1}^{N} H\left(X_{\sigma(i)} \mid X_{\sigma(1)}, \ldots, X_{\sigma(i-1)}, B\right) .
\end{aligned}
$$

Note that for a given index $j$, the number of terms of the form $H\left(X_{j} \mid \ldots, B\right)$ on the left- and righthand sides of the above inequality is equal to $N\left(\begin{array}{c}N-1 \\ K-1\end{array}\right)=M K$; however, the entropies on the left-hand side are conditioned on the same or fewer variables than the entropies on the right-hand side, therefore, the inequality (21) holds, which is equivalent to (18). Hence, by combining (17) and (18), we get

$$
K \tilde{u}_{1}+N u_{2} \leq N K,
$$

or

$$
\begin{aligned}
& \left(m_{2}-2 z\right) \tilde{u}_{1}+\left(m_{1}+m_{2}-m_{12}\right) u_{2} \\
\leq \quad & \left(m_{1}+m_{2}-m_{12}\right)\left(m_{2}-2 z\right),
\end{aligned}
$$

which after substituting $\tilde{u}_{1}=u_{1}-\left(m_{12}-m_{2}-2 z\right)$ gives

$$
\begin{aligned}
& \left(m_{2}-2 z\right) u_{1}+\left(m_{1}+m_{2}-m_{12}\right) u_{2} \\
\leq \quad & \left(m_{1}-2 z\right)\left(m_{2}-2 z\right)
\end{aligned}
$$

which matches (16).

- Case 3.

$$
\begin{aligned}
& m_{12}-m_{2}<2 z \\
& m_{12}-m_{1}<2 z .
\end{aligned}
$$

If (23) and (24) are satisfied, then (10) can be simplified as

$\left(m_{2}-2 z\right) u_{1}+\left(m_{1}-2 z\right) u_{2} \leq\left(m_{1}-2 z\right)\left(m_{2}-2 z\right)$

Suppose $m_{1} \geq m_{2}$. From (23) it follows that

$$
m_{1}-2 z<m_{1}+m_{2}-m_{12},
$$

therefore, we can construct a 3-layer network $\mathcal{G}^{\prime \prime}$ similarly to Case 2, but with $N=m_{1}-2 z, K=$ $m_{2}-2 z$ and $\tilde{u}_{1}=u_{1}$. Then by (22),

$$
K u_{1}+N u_{2} \leq N K,
$$

which matches (25).

Corollary 1: The upper-bound given by Theorem 2 is tight for any one-source two-sink 3-layer network, when both $m_{12}-m_{1}-2 z \geq 0$ and $m_{12}-m_{2}-2 z \geq 0$ or $m_{1}=m_{2}$.

Proof: When both $m_{12}-m_{1}-2 z \geq 0$ and $m_{12}-$ $m_{2}-2 z \geq 0$, the proof corresponds to Case 1 of the proof of Theorem 3. When $m_{1}=m_{2}, m_{12}-m_{1}-2 z<$ $0, m_{12}-m_{2}-2 z<0$, then $l^{P}=\left|L_{2}^{P}\right|+2 z$ solves (2)-(5) with respect to partition $P$ and by Theorem 2 
$u_{1}+u_{2} \leq m_{1}-2 z$, which matches the achievable region given by Lemma 1 .

Corollary 2: Let $\mathcal{G}_{2,2}^{3}$ be a two-source two-sink 3-layer network with sources $s_{1}, s_{2}$ and sinks $t_{1}, t_{2}$, where each $s_{i}$ is demanded by $t_{i}$ and $\mathcal{G}_{2,2}^{3}$ has no links downstream of $s_{1}$ and upstream of $t_{2}$ but not $t_{1}$, and, similarly, downstream of $s_{2}$ and upstream of $t_{1}$ but not $t_{2}$ (see Figure 1 for example). Then the $z$-error correction capacity region of $\mathcal{G}_{2,2}^{3}$ is given by (8)-(10). Moreover, it can be achieved by linear coding.

Proof: Note that the capacity region of $\mathcal{G}_{2,2}^{3}$ in the error-free case is the same as that of its related onesource two-sink 3-layer network $\mathcal{G}_{1,2}^{3}$. Thus, the achievable error-correction region of Theorem 1 for $\mathcal{G}_{2,2}^{3}$ is the same as that of $\mathcal{G}_{1,2}^{3}$, which by Theorem 3 coincides with the error-correction capacity of $\mathcal{G}_{1,2}^{3}$. Since the errorcorrection capacity region of $\mathcal{G}_{2,2}^{3}$ is upper-bounded by the error-correction capacity region of $\mathcal{G}_{1,2}^{3}$, the result follows.

Corollary 3: An outer bound on the $z$-error correction capacity region $\mathbb{U}$ of a two-sink network is given by

$$
\begin{aligned}
u_{1} & \leq m_{\mathcal{S}, t_{1}}-2 z \\
u_{2} & \leq m_{\mathcal{S}, t_{2}}-2 z \\
\mathbb{U} & \subseteq \bigcap_{P} \mathbb{V}_{1,2}^{3}(P) . \\
& \begin{array}{l}
P=\left(V_{\mathcal{S}}, V_{\mathcal{T}}\right) \\
\operatorname{cut}(P) \text { has no } \\
\text { feedback links }
\end{array}
\end{aligned}
$$

Proof: $\mathbb{U}$ is upper-bounded by $\mathbb{U}_{1,2}^{3}(P)$ for every partition $P$ such that $\operatorname{cut}(P)$ does not contain feedback links. By Theorem 3, $\mathbb{U}_{1,2}^{3}(P)=\mathbb{V}_{1,2}^{3}(P)$.

\section{ILLUSTRATIVE EXAMPLES}

Figure 4(a) depicts a one-source two-sink network topology with one feedback link across the second layer. The capacity region of this network in the error-free case is given by the cutset bounds [8], [9], [10] (see Figure 4(b)). In the presence of $z=1$ error, the cutset bound $u_{1}+u_{2} \leq 5-2=3$ is not achieved (see Figure 4(c)). By comparing the achievable region constructed using the procedure described in Section III and the upperbound $u_{1}+u_{2} \leq 2$ given by Theorem 2 , we see that in this case the upper-bound given by Theorem 2 is tight.

Figure 5(a) shows a one-source two-sink 3-layer network topology, whose capacity region in case of one network error given by the constraints $u_{1} \leq 4, u_{2} \leq 2$, $2 u_{1}+3 u_{2} \leq 8$ (as follows from Theorem 3 , shaded
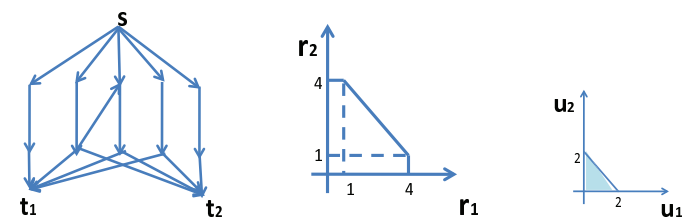

(a) One-source two (b) Error-free capac- (c) $z$-error sink network with one ity region given by correction backward link across $r_{1} \leq 4, r_{2} \leq 4, r_{1}+$ capacity region, the cut. $\quad r_{2} \leq 5 . \quad$ which coincides with the constructed achievable region of Lemma 1 and the outer bound of Theorem 2 .

Fig. 4. Example of the one-source two-sink network with backward link across the cut whose error correction capacity region is given by the upper bound Theorem 2 when $z=1$.
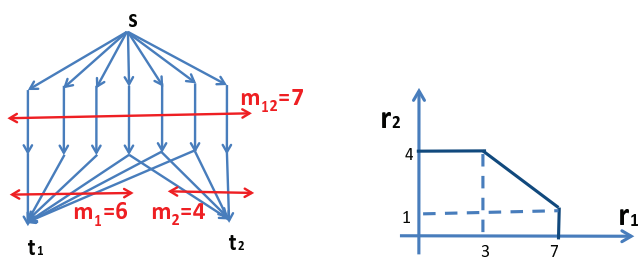

(a) One-source two-sink 3- (b) Error-free capacity relayer network with $m_{1}=6$, gion described by $r_{1} \leq$ $m_{2}=4$ and $m_{12}=7 . \quad 6, r_{2} \leq 4, r_{1}+r_{2} \leq 7$.

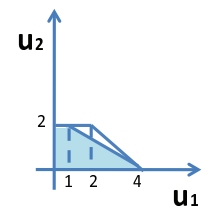

(c) Error-correction capacity region described by $u_{1} \leq 4, u_{2} \leq$ $2,2 u_{1}+3 u_{3} \leq 8$ when $z=1$ (shaded area) vs. the upper bound $u_{1}+u_{2} \leq 4$ given by Theorem 2 (unshaded area).

Fig. 5. Example of the one-source two-sink 3-layer network for which the upper bound given by Theorem 2 is not tight in case of one network error.

area in Figure 5(c)). However, as one can observe from the unshaded area in Figure 5(c), for this network the upper bound $u_{1}+u_{2} \leq 4$ given by Theorem 2 is not tight when $z=1$. 


\section{CONCLUSION}

In this paper, we derived an upper bound on the error correction capacity region for non-multicast networks, which is loose in the general case. We also found the explicit error-correction capacity region for one-source two-sink 3-layer networks, which is given by timesharing of the extreme points, and used it to refine the obtained upper bound for general two-sink networks.

\section{ACKNOWLEDGMENT}

We thank Michelle Effros, Michael Langberg, Joerg Kliewer and Sid Jaggi for interesting and helpful discussions.

\section{REFERENCES}

[1] R. W. Yeung and N. Cai, "Network error correction, part I: Basic concepts and upper bounds," Commun. Inf. Syst., vol. 6, no. 1, pp. 19-36, 2006.

[2] S. Vyetrenko, T. Ho, M. Effros, J. Kliewer, and E. Erez, "Rate rregions for coherent and noncoherent multisource network error correction," June 2009, pp. $1001-1005$.

[3] S. Jaggi, M. Langberg, S. Katti, T. Ho, D. Katabi, M. Médard, and M. Effros, "Resilient network coding in the presence of byzantine adversaries," Information Theory, IEEE Transactions on, vol. 54, no. 6, pp. $2596-2603$, June 2008.

[4] R. Koetter and F. R. Kschischang, "Coding for errors and erasures in random network coding," IEEE Transactions on Information Theory, August 2008.

[5] H. Yao, T. K. Dikaliotis, S. Jaggi, and T. Ho, "Multi-source operator channels: Efficient capacity-achieving codes," August 2010.

[6] T. Chan and A. Grant, "Mission impossible: Computing the network coding capacity region," July 2008, pp. $320-324$.

[7] N. Harvey and R. Kleinberg, "Tighter cut-based bounds for kpairs communication problems," in Proc. 43rd Allerton Conference on Communication, Control, and Computing, Monticello, IL, September 2005.

[8] E. Erez and M. Feder, "Capacity region and network codes for two receivers multicast with private and common data," 2003.

[9] C. Ngai and R. Yeung, "Multisource network coding with two sinks," vol. 1, June 2004, pp. 34 - 37.

[10] A. Ramamoorthy and R. D. Wesel, "The single source two terminal network with network coding," in in Canadian Workshop on Information Theory, 2005.

[11] R. Koetter and M. Médard, "An algebraic approach to network coding," IEEE/ACM Transactions on Networking, vol. 11, no. 5, pp. 782-795, October 2003.

[12] S. Kim, T. Ho, M. Effros, and S. Avestimehr, "Network error correction with unequal link capacities," in Proc. of the 47th annual Allerton conference on Communication, control, and computing, September 2009, pp. 1387-1394.

[13] O. Kosut, L. Tong, , and D. Tse, "Nonlinear network coding is necessary to combat general byzantine attacks," in Proc. of the 47th annual Allerton conference on Communication, control, and computing, September 2009, pp. 593 - 599. 